\title{
On the Surface Area of the Ellipsoid*
}

\author{
By Stuart R. Keller
}

\begin{abstract}
An expression is derived for the surface area of an ellipsoid in the form of a convergent series. The derivation is based upon an $n$-point Gauss-Chebyshev numerical quadrature. The rate of convergence and accuracy of the formula are demonstrated by computing the surface area of several ellipsoids.
\end{abstract}

1. Introduction. While the ellipsoid is a very useful three-dimensional geometric shape, it suffers from an annoying peccadillo. Except for the special cases of the sphere, the prolate spheroid, and the oblate spheroid, no closed form expression exists for the surface area of the ellipsoid. This situation arises because of the fact that it is impossible to carry out the integration in the expression for the surface area in closed form for the most general case of three unequal axes.

In spite of the widespread use of the ellipsoid as a mathematical model and the existence of an enormous body of knowledge on the theory of curved surfaces and numerical integration, it does not appear that the problem of approximating the surface area of a triaxial ellipsoid has been addressed.

In this paper an expression is derived for the surface area of an ellipsoid in the form of a convergent series. The derivation is based upon an $n$-point Gauss-Chebyshev numerical quadrature. The rate of convergence and accuracy of the formula are demonstrated by computing the surface area of several ellipsoids.

2. Derivation. The equation of an ellipsoidal surface having semiaxis lengths $a, b$, and $c$ may be written as

$$
z=f(x, y)= \pm c\left(1-x^{2} / a^{2}-y^{2} / b^{2}\right)^{1 / 2} \quad \text { for } x^{2} / a^{2}+y^{2} / b^{2} \leqslant 1 .
$$

An element of surface area is given by

$$
d S=\left[1+f_{x}^{2}+f_{y}^{2}\right]^{1 / 2} d x d y
$$

The surface area may be written as

$$
S=4 \int_{0}^{a} G(x) d x,
$$

where

$$
G(x)=\int_{-b\left(1-x^{2} / a^{2}\right)^{1 / 2}}^{b\left(1-x^{2} / a^{2}\right)^{1 / 2}} \frac{\left[1-\left(1-c^{2} / a^{2}\right) x^{2} / a^{2}-\left(1-c^{2} / b^{2}\right) y^{2} / b^{2}\right]^{1 / 2}}{\left[1-x^{2} / a^{2}-y^{2} / b^{2}\right]^{1 / 2}} d y .
$$

Received March 3, 1978.

AMS (MOS) subject classifications (1970). Primary 53A05; Secondary 65D 30.

* This research was sponsored in part by NIH grant HL-16851. 
In order that $G(x)$, the inner integral, may be evaluated effectively, it is desirable that it be written in such a manner that a numerical integration rule of Gauss type (Davis and Rabinowitz [1, p. 35]) may be employed. Therefore, a change of variable of integration is made via

$$
t=\frac{y}{b\left(1-x^{2} / a^{2}\right)^{1 / 2}} .
$$

Then $G(x)$ may be written as

$$
G(x)=\int_{-1}^{1} \frac{g(x, t)}{\sqrt{1-t^{2}}} d t
$$

where

(7) $g(x, t)=b\left\{\left[1-\left(1-c^{2} / b^{2}\right) t^{2}\right]-\left[\left(1-c^{2} / a^{2}\right)-\left(1-c^{2} / b^{2}\right) t^{2}\right] x^{2} / a^{2}\right\}^{1 / 2}$.

This formulation of $G(x)$ makes it amenable to $n$-point Gauss-Chebyshev numerical integration (Davis and Rabinowitz [1, pp. 34, 75]):

$$
G(x) \approx \frac{\pi}{n} \sum_{j=1}^{n} g\left(x, t_{j}\right)
$$

where

$$
t_{j}=\cos \left(\frac{2 j-1}{2 n} \pi\right), \quad j=1,2, \ldots, n
$$

Therefore, the surface area may be approximated by

$$
S \approx 4 \int_{0}^{a}\left\{\frac{\pi}{n} \sum_{j=1}^{n} g\left(x, t_{j}\right)\right\} d x .
$$

Since the order of integration and summation may be interchanged, Eq. (10) may be written as

$$
S \approx \frac{4 \pi}{n} \sum_{j=1}^{n} \int_{0}^{a} g\left(x, t_{j}\right) d x .
$$

The integral in this expression may be written as

$$
I=\int_{0}^{a} g\left(x, t_{j}\right) d x=b \int_{0}^{a}\left(\alpha+\beta x^{2}\right)^{1 / 2} d x,
$$

where

$$
\alpha=1-\left(1-c^{2} / b^{2}\right) t_{j}^{2}
$$

and

$$
\beta=-\left[\left(1-c^{2} / a^{2}\right)-\left(1-c^{2} / b^{2}\right) t_{j}^{2}\right] / a^{2} .
$$

This integral may be evaluated using well-known formulas. 
If $a=b=c$ :

$$
\begin{aligned}
& \alpha=1, \\
& \beta=0,
\end{aligned}
$$

and

$$
I=a^{2} .
$$

Therefore, for the surface area of the sphere, Eq. (11) reduces to the exact result

$$
S=4 \pi a^{2} \text {. }
$$

If $a>b \geqslant c$ or $a \geqslant b>c:$

$$
\begin{aligned}
& \alpha>0, \\
& \beta<0,
\end{aligned}
$$

and

$$
I=(b / 2)\left\{a\left(\alpha+\beta a^{2}\right)^{1 / 2}+\left[\alpha /(-\beta)^{1 / 2}\right] \sin ^{-1}\left[a(-\beta / \alpha)^{1 / 2}\right]\right\}
$$

Therefore,

$$
\begin{gathered}
S \approx \frac{2 \pi a b}{n} \sum_{j=1}^{n}\left\{\frac{c}{a}+\frac{1-\left(1-c^{2} / b^{2}\right) t_{j}^{2}}{\left[\left(1-c^{2} / a^{2}\right)-\left(1-c^{2} / b^{2}\right) t_{j}^{2}\right]^{1 / 2}}\right. \\
\left.\cdot \sin ^{-1} \frac{\left[\left(1-c^{2} / a^{2}\right)-\left(1-c^{2} / b^{2}\right) t_{j}^{2}\right]^{1 / 2}}{\left[1-\left(1-c^{2} / b^{2}\right) t_{j}^{2}\right]^{1 / 2}}\right\} .
\end{gathered}
$$

Since Gaussian rules (such as Eq. (8)) have the property that they converge to the exact integral as $n \rightarrow \infty$ for continuous integrands (Davis and Rabinowitz [1, p. 48]), it is clear that Eq. (22) will converge to the exact surface area as $n \rightarrow \infty$. Furthermore, for the case of a prolate spheroid $(a>b=c)$, Eq. (22) reduces to

$$
S=2 \pi b^{2}+\frac{2 \pi a b}{\left(1-b^{2} / a^{2}\right)^{1 / 2}} \sin ^{-1}\left(1-b^{2} / a^{2}\right)^{1 / 2},
$$

which is the well-known exact result (see e.g. Standard Mathematical Tables [2, p. 496]).

3. Examples. In order to illustrate the rate of convergence and accuracy of Eq. (22), several examples have been computed. In carrying out the actual computations the number of terms necessary to compute the sum in Eq. (22) for any given $n$ may be reduced by noting that the expression within the brackets is an even function of $t_{j}$ and $t_{j}=-t_{n-j+1}$. Therefore, Eq. (22) may be written as

$$
S \approx 2 \pi b c+\frac{4 \pi a b}{n} \sum_{j=1}^{n / 2} s\left(a, b, c, t_{j}\right) \quad \text { if } n \text { is even }
$$


or

(25) $S \approx 2 \pi b c+\frac{2 \pi a b}{n} s(a, b, c, 0)+\frac{4 \pi a b}{n} \sum_{j=1}^{(n-1) / 2} s\left(a, b, c, t_{j}\right)$ if $n$ is odd,

where

(26)

$$
s\left(a, b, c, t_{j}\right)=\frac{1-\left(1-c^{2} / b^{2}\right) t_{j}^{2}}{\left[\left(1-c^{2} / a^{2}\right)-\left(1-c^{2} / b^{2}\right) t_{j}^{2}\right]^{1 / 2}}
$$

$$
\cdot \sin ^{-1} \frac{\left[\left(1-c^{2} / a^{2}\right)-\left(1-c^{2} / b^{2}\right) t_{j}^{2}\right]^{1 / 2}}{\left[1-\left(1-c^{2} / b^{2}\right) t_{j}^{2}\right]^{1 / 2}} \text {. }
$$

For $n=1$, the last term in Eq. (25) would of course be omitted.

\section{TABLE 1}

Surface area of some ellipsoids

computed from Eqs. (24) and (25)

\begin{tabular}{|c|c|c|c|c|c|}
\hline \multicolumn{2}{|c|}{$a=1, b=1, c=0.5$} & \multicolumn{2}{|c|}{$a=1, b=0.8, c=0.625$} & \multicolumn{2}{|c|}{$\mathrm{a}=2, \mathrm{~b}=1, \mathrm{c}=0.25$} \\
\hline & $\begin{array}{c}\text { surface } \\
\text { area }\end{array}$ & & $\begin{array}{c}\text { surface } \\
\text { area }\end{array}$ & & $\begin{array}{c}\text { surface } \\
\text { area }\end{array}$ \\
\hline $\mathrm{n}$ & $S$ & $\mathrm{n}$ & $\mathrm{S}$ & $\mathrm{n}$ & $S$ \\
\hline 1 & 10.73921766 & 1 & 8.908899289 & 1 & 19.87868264 \\
\hline 2 & 8.823782978 & 2 & 8.171982315 & 2 & 14.57211620 \\
\hline 3 & 8.694757583 & 3 & 8.152738902 & 3 & 13.95444232 \\
\hline 4 & 8.676245214 & 4 & 8.151697468 & 4 & 13.79280116 \\
\hline 5 & 8.672825475 & 5 & 8.151625183 & 5 & 13.73763071 \\
\hline 6 & 8.672103135 & 6 & 8.151619464 & 6 & 13.71610159 \\
\hline 7 & 8.671937123 & 7 & 8.151618973 & 7 & 13.70699092 \\
\hline 8 & 8.671896685 & 8 & 8.151618929 & 8 & 13.70291858 \\
\hline 9 & 8.671886407 & 9 & 8.151618925 & 9 & 13.70102459 \\
\hline 10 & 8.671883709 & 10 & 8.151618924 & 10 & 13.70011667 \\
\hline 20 & 8.671882705 & 20 & 8.151618924 & 20 & 13.69921242 \\
\hline 30 & 8.671882705 & 30 & 8.151618924 & 30 & 13.69921081 \\
\hline 40 & 8.671882705 & 40 & 8.151618924 & 40 & 13.69921081 \\
\hline $\begin{array}{l}\text { exact } \\
\text { value }\end{array}$ & 8.671882703 & & & & \\
\hline
\end{tabular}

Semiaxis Lengths

The results for three ellipsoids having semiaxes ( $a=1, b=1, c=0.5),(a=1$, $b=0.8, c=0.625)$, and $(a=2, b=1, c=0.25)$ are shown in Table 1. Since the volume of an ellipsoid is given by $4 \pi a b c / 3$, each of these ellipsoids has the same volume. The exact value for the surface area of the first ellipsoid, which is an oblate spheroid $(a=b>c)$, was calculated from the well-known exact result (see e.g. Standard Mathematical Tables [2, p. 495]): 


$$
S=2 \pi a^{2}+\frac{\pi c^{2}}{\left(1-c^{2} / a^{2}\right)^{1 / 2}} \ln \frac{1+\left(1-c^{2} / a^{2}\right)^{1 / 2}}{1-\left(1-c^{2} / a^{2}\right)^{1 / 2}} .
$$

It is seen that for the first two ellipsoids, Eqs. (24) and (25) are accurate to at least 10 digits when $n=20$. For the third case, which corresponds to a very "eccentric" ellipsoid, the rate of convergence is slower as this degree of accuracy was not obtained until $n=30$.

4. Concluding Remarks. Surface integrals of many functions over the ellipsoidal surface may be carried out in a manner analogous to the above with an appropriate redefinition of the function $g(x, t)$. This may require, however, that the integration over $x$ be carried out numerically rather than exactly as was done in the present case.

Department of Civil Engineering and Engineering Mechanics

Columbia University

New York, New York 10027

1. P. J. DAVIS \& P. RABINOWITZ, Numerical Integration, Blaisdell, Waltham, Mass., 1967. MR 35 \#2482.

2. Standard Mathematical Tables, 14 th ed. (S. M. Selby, Editor), The Chemical Rubber Co., Cleveland, Ohio, 1965. 